\title{
Podocyte loss and albuminuria of KK-Ay mouse: A spontaneous animal model for human type 2 diabetic nephropathy*
}

\author{
Yuji Ishikawa, Takamichi Ito, MitsuoTanimoto, Shinji Hagiwara, Masako Furukawa, \\ Saori Yamaguchi, Keisuke Omote, Katsuhiko Asanuma, Tomohito Gohda, \\ Yoshio Shimizu, Kazuhiko Funabiki, Satoshi Horikoshi, Yasuhiko Tomino\# \\ Division of Nephrology, Department of Internal Medicine, Faculty of Medicine, Juntendo University, Tokyo, Japan; \\ \#Corresponding Author: yasu@juntendo.ac.jp
}

Received 17 April 2012; revised 16 May 2012; accepted 27 May 2012

\begin{abstract}
Podocyte loss was well known in type 2 diabetic nephropathy patients. The objective of the present study was to determine the number of podocytes and the degree of albuminuria in diabetic $K K-A^{y} / T a\left(K K-A^{y}\right)$ mice which had been reported as diabetic nephropathy model. Diabetic KK- $A^{y}$ mice, diabetic KK/Ta mice and nondiabetic BALB/CA Jcl (BALB/CA) mice were studied. We analyzed glomerular lesions in all mice by morphometric analysis and immunofluorescence to determine the number of podocytes. Level of urinary albumin was also measured. Glomerular enlargement and mesangial expansion were observed in $\mathrm{KK}-\mathrm{A}^{y}$ mice. Mean number of podocytes per glomerulus (NG pod) in diabetic $K K-A^{y}$ mice was significantly lower than that in non-diabetic BALB/CA mice. Mean NG pod/glomerular area (GA) per glomerulus was also significantly decreased in diabetic $K K-\mathrm{A}^{y}$ mice. The level of urinary albumin/creatinine ratio (ACR) in diabetic KK- $A^{y}$ mice was significantly higher than that in non-diabetic BALB/CA mice. These data suggest that podocyte loss might induce albuminuria in KK-A mice. This finding confirmed our previous report that KK-A mice, especially in terms of histological findings, are a suitable animal model for glomerular injury in type 2 diabetic nephropathy.
\end{abstract}

Keywords: Podocyte Loss; Albuminuria; Diabetic Rodent Model; Diabetes Type 2; Diabetic Nephropathy

\section{INTRODUCTION}

Morphometric analysis has contributed greatly to our

${ }^{*}$ Conflicts of interest: None declared. understanding of diabetic nephropathy [1-3]. Generally, the progression of nephropathy was associated with a reduction in the number of podocytes per glomerulus. Remaining podocytes were obligated to grow and to extend their foot processes to maintain the area covered. We previously reported that podocyte injury might provide additional prognostic information in patients with IgA nephropathy, the most common chronic glomerulonephritis, using a small amount of renal biopsy tissue [4]. Lemley et al. [5] reported podocytopenia (podocyte loss) and disease activity in patients with IgA nephropathy. However, glomerular structure in type 2 diabetes has been studied less extensively although this form of diabetes is a more common cause of end stage kidney disease. Macedo et al. [6] reported that the control of hyperglycemia prevented glomerular basement membrane (GBM) thickening in early and late (12 months) alloxan diabetic nephropathy and podocyte number reduction.

The KK/Ta mouse, a model of type 2 diabetic nephropathy, was established from Japanese native mice as an inbred mouse by Kondo et al. in 1957 [7]. This mouse is generally considered to be a polygenic disease model. Male KK/Ta mice spontaneously exhibit type 2 diabetes associated with hyperglycemia, glucose intolerance, hyperinsulinemia, mild obesity and microalbuminuria more severe than those in the female [8-10]. KK-A $\mathrm{A}^{y}$ mice showed obesity, hyperglycemia including high levels of $\mathrm{HbA1c}$ and albuminuria [11]. The pathological changes of glomeruli in KK- $\mathrm{A}^{y}$ mice were consistent with those in the early stage of human diabetic nephropathy. The renal histological changes in $\mathrm{KK}-\mathrm{A}^{y}$ mice are more severe than those in $\mathrm{KK} / \mathrm{Ta}$ mice. Advanced glycation end products (AGE) and transforming growth factor-beta (TGF-b) protein appeared to be localized in the glomerular mesangial areas [11]. It appears that KK- $\mathrm{A}^{y}$ mice, especially in terms of histopathological findings, are a suitable animal model for the early stage of type 2 diabetic nephropathy. 
The objective of the present study was to determine the number of podocytes and the degree of albuminuria in $\mathrm{KK}-\mathrm{A}^{y}$ mice.

\section{MATERIALS AND METHODS}

\subsection{Animals}

Male diabetic KK-A $\mathrm{A}^{y} / \mathrm{Ta}\left(\mathrm{KK}-\mathrm{A}^{y}\right)$ mice, male diabetic $\mathrm{KK} / \mathrm{Ta} \mathrm{Jcl}(\mathrm{KK} / \mathrm{Ta})$ mice and male non-diabetic BALB/ cA Jcl (BALB/cA) mice at 20 weeks after birth were obtained from CLEA Japan Inc. (Tokyo, Japan). Mice were housed individually in air-conditioned specific pathogen-free (SPF) animal quarters kept at $24^{\circ} \mathrm{C} \pm 1{ }^{\circ} \mathrm{C}$ with lighting from 8:00 AM to 9:00 PM, and given unrestricted access to special food (rodent pellet diet NMF; $348 \mathrm{kcal} / 100 \mathrm{~g}$, containing 5.5\% crude fat, CLEA Japan Inc., Tokyo, Japan) and water throughout the experimenttal period of 20 weeks. All animals received humane care in compliance with the experimental protocol approved by the Committee of Laboratory Animals according to institutional guidelines.

\subsection{Biochemical Findings}

At 8, 12, 16 and 20 weeks of age, body weight (BW) was checked, and urinary albumin/creatinine ratio (ACR) and hemoglobin A1c (HbA1c) were measured. At 8 and 20 weeks of age, blood pressure was checked by a noninvasive tail cuff and pulse transducer system (Softron BP-98A, Tokyo, Japan) at 11:00 AM after the mice were externally prewarmed at $38^{\circ} \mathrm{C}$ for $10 \mathrm{~min}$. After repetition of each measurement (from 3 to 6 times), standard deviations of less than 10.0 were defined as the blood pressure levels. At the same weeks of age, levels of fasting and casual blood glucose were measured.

Metabolic cages obtained from CLEA Japan Inc. (Tokyo, Japan) were used for collecting of urinary samples for 24 hours. To measure urinary albumin and creatinine at $8,12,16$ and 20 weeks of age, an immunoassay was performed using a commercial kit (DCA 2000 system, Bayer Diagnostics, Elkhart, IN, USA) [12]. Glucose taken from the retro-orbital sinus was measured using a commercial kit (Glucocard; Kyoto Daiichi Kagaku, Kyoto, Japan).

\subsection{Morphometric Analysis by Light and Immunofluorescent Microscopy}

Mice of all groups were sacrificed by exsanguination from the inferior vena cava at 20 weeks and the kidneys were perfused by saline via the left ventricle for $5 \mathrm{~min}$ at a pressure of $150 \mathrm{mmHg}$ without prior flushing of the vasculature. Renal, urine and serum samples were kept frozen at $-80^{\circ} \mathrm{C}$ until assayed. Renal tissues of these mice fixed in $20 \%$ formaldehyde were embedded in paraffin and cut serially to obtain 3 -um thick sections. All sections were mounted on glass slides. Formalin-fixed, paraffin-embedded renal sections were deparaffinized, and stained with periodic-acid Schiff (PAS).

Immunofluorescent staining for podocytes in renal tissues was performed using the polyclonal rabbit antimouse WT1 antibody (IgG) (Santa Cruz, SC-192). Cryostat $3 \mu \mathrm{m}$ renal sections were air-dried for $10 \mathrm{~min}$ and fixed in cold acetone for $10 \mathrm{~min}$. To reduce the background, the non-specific binding was blocked by incubating with blocking solution (as above) for $30 \mathrm{~min}$. Non-specific staining was blocked by incubation for 15 min with avidin and biotin using the avidin-biotin blocking kit (Vector Laboratories). The sections were then incubated with the primary antibody $(\mathrm{Ab})$ diluted 1:25 in the blocking solution at room temperature for $60 \mathrm{~min}$. The primary $\mathrm{Ab}$ used was polyclonal rabbit anti-mouse WT-1 antibody (IgG) (Santa Cruz, SC-192). The sections were then incubated at room temperature for $30 \mathrm{~min}$. Goat anti-rabbit Alexa Fluor 488 was used to visualize WT-1 positive cells (podocytes). The sections were mounted with a fluorescent mounting media (Dako Cytomation) before visualization with a Fluoview 1000 confocal microscope (Olympus, Tokyo, Japan) and FV10-ASWsoftware (version 1.3c; Olympus).

Sixty one, 70 and 90 midsections of glomeruli taken from KK-A ${ }^{y}, \mathrm{KK} / \mathrm{Ta}$ and BALB/cA mice, respectively, were used in this study. Renal specimens were observed and photographed using a microscope equipped with a digital imaging system (Leica DM 2000, Leica Microsystems GmbH., Germany). The prints of each glomerulus in a $\times 400$ field on each specimen were used. In the section, the glomerular tufts were carefully traced by hands with a pencil marker and prepared for the analysis. Morphometrical analysis was performed using a KS-400 version 4.0 image analysis system (KS-400, Carl Zeiss Vision, Munich, Germany). The number of podocytes per glomerulus (NG pod) and glomerular area (GA) were determined using the method of Weibel [13]. Ratio of NG pod to GA (NG pod/GA) was also calculated. Specimens with glomerular lesions such as sclerosis, adhesion and/or crescents in $\mathrm{KK}-\mathrm{A}^{y}$ mice at 20 weeks of age were excluded from the calculation of podocytes. These examinations were performed by two investigators without knowledge of the origin of the slides and then mean values were calculated.

\subsection{Detection of Apoptotic Cell in Glomerulus by TUNEL Staining}

Apoptosis in renal sections was determined by TUNEL assay using the ApopTag in situ apoptosis detection kit (S7101, Chemicon International, Temecula, CA). In brief, $6 \mu \mathrm{m}$ paraffin-embedded renal sections were deparaffinized with xylene, blocked with $3 \% \mathrm{H}_{2} \mathrm{O}_{2}$ for $10 \mathrm{~min}$, 
and then washed with PBS. Terminal deoxynucleotidyl transferase (TdT) and biotin-11-dUTP were supplemented followed by incubation for $60 \mathrm{~min}$. Then, the sections were washed with PBS, incubated with avidinbiotinylated horseradish peroxidase complex followed by color development with DAB. Counter-staining for nuclei, dehydration, clearing and mounting were performed. Cells with brown granules in nuclei were TUNEL positive cells and TUNEL positive podocytes on the GBM were counted in 30 glomeruli of transverse section. Results were expressed as average numbers of TUNELpositive podocytes per glomerulus.

\subsection{Statistical Analysis}

Data were expressed as mean $\pm \mathrm{SD}$ or mean $\pm \mathrm{SE}$. Statistical differences between means were determined using analysis of variance (ANOVA) on ranks followed by a post hoc test (Student-Newman-Keuls all pairwise comparison procedures). Statistical significance of \% survival was determined by the log-rank test. A value of $p<$ 0.05 was specified before the study to indicate significance.

\section{RESULTS}

\subsection{Phenotypes}

Levels of urinary albumin/creatinine ratio (ACR) of diabetic KK- $\mathrm{A}^{\mathrm{y}}$ mice were significantly higher than those of non-diabetic BALB/cA mice and $\mathrm{KK} / \mathrm{Ta}$ mice at all time points $(\mathrm{p}<0.001)$ (Figure 1). The levels of BW in all strains mice increased gradually after 8 weeks of age. These levels in KK- $\mathrm{A}^{y}$ mice were significantly higher than those in BALB/cA mice and KK/Ta mice $(\mathrm{p}<0.001)$ (Table 1). The levels of HbAlc in KK-A ${ }^{y}$ mice were also significantly higher than those in BALB/cA mice and KK/Ta mice $(p<0.001)$. The levels of fasting blood glucose in KK-A $\mathrm{A}^{y}$ mice were did not change among three strains. No significant changes were observed in the levels of systolic, diastolic and mean blood pressure among three strains mice at 8 and 20 weeks of age (Table 1).

\subsection{Glomerular Changes}

In light microscopy, diffuse expansion of glomerular mesangial matrices was observed in diabetic KK- $\mathrm{A}^{y}$ mice (Figure 2). Sclerosed lobules with several homogenous PAS-positive hyaline caps were found at 20 weeks of age.

\subsection{Number of Podocytes}

Mean podocyte numbers per glomerulus (NG pod) in diabetic KK-A $\mathrm{A}^{y}$ mice (mean $\pm \mathrm{SD}: 8.73 \pm 2.54$ ) and $\mathrm{KK} / \mathrm{Ta}$ mice (mean $\pm \mathrm{SD}: 7.84 \pm 1.93$ ) were significantly

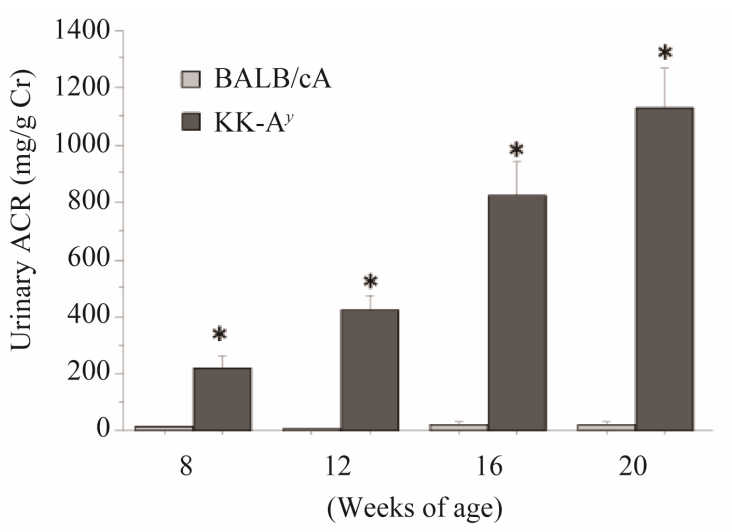

$* \mathrm{p}<0.001$ vs BALB/cA mice

Figure 1. Mean level of urinary albumin/creatinine ratio (ACR) in $\mathrm{KK}_{-} \mathrm{A}^{y}$ mice $(\boldsymbol{\square}, \mathrm{n}=10)$ and BALB/cA mice $(\square, \mathrm{n}=10)$. Date expressed as means \pm S.E.
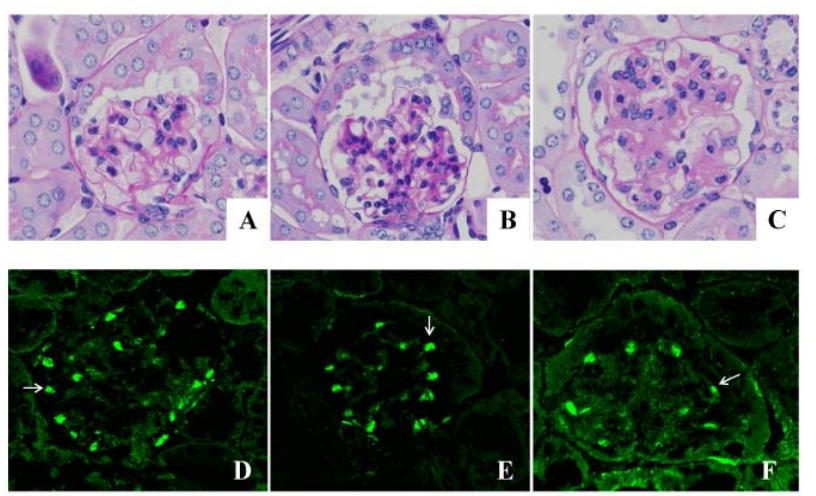

Figure 2. Light microscopy of renal tissue section (A,B,C). Diffuse expansion of glomerular mesangial matrices was observed in KK/Ta mice (B) and KK- $\mathrm{A}^{y}$ mice (C). Sclerosed lobules with several homogenous periodic acid-Schiff (PAS)positive hyaline caps were observed at 20 weeks of age (C). Immunofluorescent staining for WT-1 positive cells (podocytes) in the renal sections (D, E, F). WT-1 positive cells (podocytes) in $\mathrm{KK} / \mathrm{Ta}$ mice and $\mathrm{KK}-\mathrm{A}^{y}$ mice were fewer than those in $\mathrm{BALB} / \mathrm{cA}$ mice. Original magnification, $\times 400$. (A, D) BALB/ cA mouse at 20 weeks of age. (B, E) KK/Ta mouse at 20 weeks of age. (C, F) KK-A $\mathrm{A}^{y}$ mouse at 20 weeks of age.

lower than those in non-diabetic BALB/cA mice (mean \pm SD: $9.47 \pm 2.58)\left(K K-A^{y}\right.$ vs BALB/cA: $p<0.05, \mathrm{KK} / \mathrm{Ta}$ vs BALB/cA: p < 0.01) (Figure 3(a)).

A significant change in the glomerular area (GA) was observed among diabetic KK-A $\mathrm{A}^{y}$ mice (mean $\pm \mathrm{SD}$ : $5924.8 \pm 1846.4$ ), KK/Ta mice (mean \pm SD: $4372.7 \pm$ 1232.3) and non-diabetic BALB/cA mice (mean \pm SD: $3738 \pm 1338.4)\left(\mathrm{KK}_{-} \mathrm{A}^{y}\right.$ vs BALB/cA: $\mathrm{p}<0.001, \mathrm{KK} / \mathrm{Ta}$ vs BALB/cA: p < 0.01) (Figure 3(b)). Mean number of podocytes per $1,000 \mu \mathrm{m}^{2}$ of glomerular area (GA) in diabetic KK-A ${ }^{\mathrm{y}}$ mice (mean $\left.\pm \mathrm{SD}: 1.6 \pm 0.6\right)$ and $\mathrm{KK} / \mathrm{Ta}$ mice (mean $\pm \mathrm{SD}: 1.9 \pm 0.6$ ) were significantly lower than that in non-diabetic BALB/cA mice (mean \pm SD: $2.7 \pm 0.9)(\mathrm{p}<0.001)($ Figure 3(c)). 
Table 1. Biochemical parameters BALB/cA, KK-Ta and KK-A mice.

\begin{tabular}{|c|c|c|c|c|}
\hline & & $\mathrm{BALB} / \mathrm{cA}$ & $\mathrm{KK}-\mathrm{Ta}$ & $\mathrm{KK}-\mathrm{A}^{y}$ \\
\hline & & $(\mathrm{n}=10)$ & $(\mathrm{n}=10)$ & $(\mathrm{n}=10)$ \\
\hline Fasting & 8 weeks & $19.1 \pm 0.2$ & $25.7 \pm 0.2^{* *}$ & $33.7 \pm 0.6^{* *}$ \\
\hline weight & 16 weeks & $21.3 \pm 0.4$ & $33.3 \pm 0.5^{* *}$ & $42.9 \pm 0.7^{* *}$ \\
\hline \multirow[t]{2}{*}{ (g) } & 20 weeks & $24.5 \pm 0.2$ & $36.7 \pm 0.5^{* *}$ & $45.1 \pm 0.7^{* *}$ \\
\hline & 8 weeks & $2.5 \pm 0.0$ & $4.1 \pm 0.1^{*}$ & $5.2 \pm 0.1^{* *}$ \\
\hline $\mathrm{HbA} 1 \mathrm{c}$ & 12 weeks & $2.5 \pm 0.0$ & $4.0 \pm 0.0^{*}$ & $7.3 \pm 0.2^{* *}$ \\
\hline \multirow[t]{2}{*}{$(\%)$} & 16 weeks & $2.5 \pm 0.0$ & $4.1 \pm 0.1^{*}$ & $8.4 \pm 0.3^{* *}$ \\
\hline & 20 weeks & $2.5 \pm 0.0$ & $4.4 \pm 0.1^{*}$ & $8.2 \pm 0.3^{* *}$ \\
\hline \multicolumn{5}{|l|}{ Fasting } \\
\hline blood & 8 weeks & $78.4 \pm 2.1$ & $82.4 \pm 6.9$ & $87.9 \pm 4.6$ \\
\hline $\begin{array}{l}\text { glucose } \\
(\mathrm{mg} / \mathrm{dl})\end{array}$ & 20 weeks & $84.7 \pm 3.3$ & $86.6 \pm 4.6$ & $85.9 \pm 5.6$ \\
\hline blood & 8 weeks & $112.7 \pm 2.7$ & $113.6 \pm 2.4$ & $114.7 \pm 2.7$ \\
\hline $\begin{array}{l}\text { pressure } \\
(\mathrm{mmHg})\end{array}$ & 20 weeks & $134.0 \pm 2.0$ & $127.2 \pm 5.0$ & $122.8 \pm 4.3$ \\
\hline
\end{tabular}

${ }^{*} \mathrm{p}<0.01$ vs BALB/cA; Data expressed as mean $\pm \mathrm{SE} ;{ }^{* *} \mathrm{p}<0.001$ vs BALB/cA.

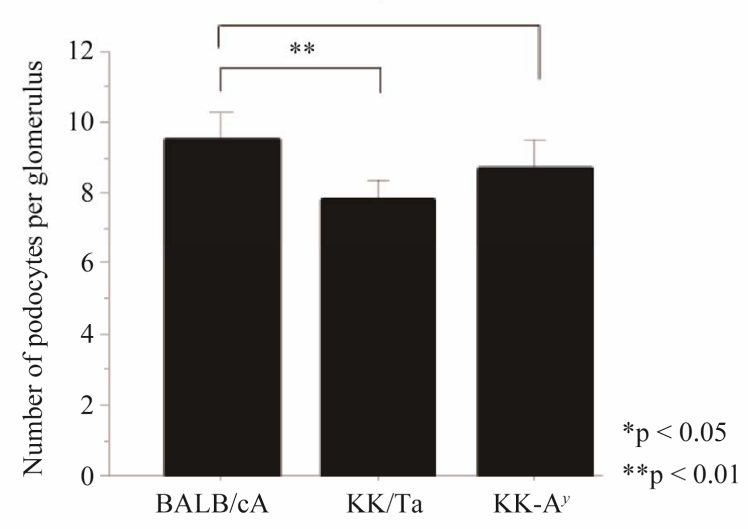

(a)

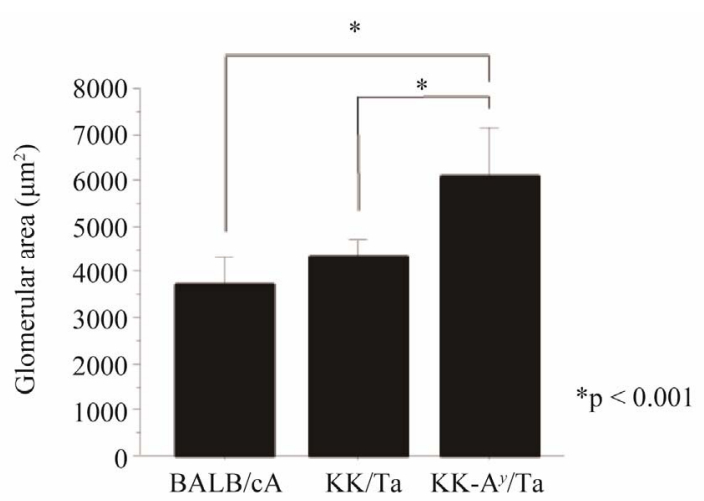

(b)

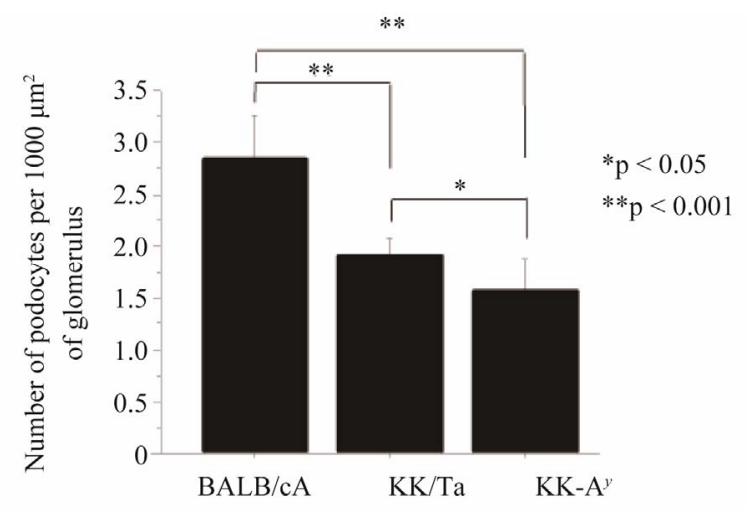

(c)

Figure 3. (a) Mean podocyte number per glomerulus (NG pod) of diabetic KK-A $\mathrm{A}^{y}$ mice was significantly lower than that of nondiabetic BALB/cA mice $\left(\mathrm{KK}-\mathrm{A}^{y}\right.$ vs BALB/cA: $\left.\mathrm{p}<0.05\right)$. At the same time, that of KK/Ta mice was significantly lower than that of non-diabetic BALB/cA mice (KK/Ta vs BALB/cA: p < 0.01); (b) The glomerular area (GA) of diabetic KK-A mice was signifycantly larger than that of BALB/cA mice $\left(K K-A^{y}\right.$ vs BALB: $\left.p<0.001\right)$. The same change was difference between KK/Ta mice and non-diabetic BALB/cA mice (KK/Ta vs BALB: $\mathrm{p}<0.001$ ); (c) The mean number of podocytes per $1000 \mu^{2}$ of glomerulus showed obvious difference between the two kinds of diabetic mice $\left(\mathrm{KK}-\mathrm{A}^{y}\right.$ and KK/Ta) and non-diabetic BALB/cA mice $(\mathrm{p}<0.001)$. 


\subsection{TUNEL Staining}

TUNEL staining was performed to detect the number of apoptotic cells per glomerulus in KK- $\mathrm{A}^{y}$ mice. An apoptotic cell was observed only one out of 30 glomeruli in KK-A $\mathrm{A}^{y}$ mice (Figure 4).

\section{DISCUSSION}

In human diabetic nephropathy, the common pathological features are glomerular enlargement, mesangial matrix expansion, segmental nodular sclerosis and glomerular basement membrane (GBM) thickening associated with albuminuria. AGE and TGF-b products have been implicated in the pathogenesis of human diabetic nephropathy $[14,15]$. Recently, we reported that KK- $\mathrm{A}^{y}$ mice exhibited type 2 diabetes and diabetic nephropathy from 8 to 20 weeks of age [11]. In PAS and PAM staining of specimens from KK-A ${ }^{y}$ mice, we observed segmental sclerosis in some glomeruli at 20 weeks of age. In immunohistochemistry, AGE and TGF-b were marked in the glomerular mesangial areas [11]. The findings for albuminuria and GBM thickening in KK- ${ }^{y}$ mice were consistent with the duration of diabetes. It was clear that the KK-A $\mathrm{A}^{y}$ mouse might be a useful model of the early stage of type 2 diabetic nephropathy [11]. Pagtalunan et al. [16] reported that GBM thickness was increased and podocyte foot processes were broadened. Broadening of podocyte foot processes was associated with a reduction in the number per glomerulus and an increase in the surface area covered by remaining podocytes from Pima Indians with type 2 diabetes. Madedo et al. [5] reported that the control of hyperglycemia prevented GBM thickening in early and late (12 months) alloxan induced type 1 diabetic nephropathy and podocyte number reduction. In this study, KK-A $\mathrm{A}^{y}$ mice at 20 weeks of age showed diffuse expansion of glomerular mesangial matrices with

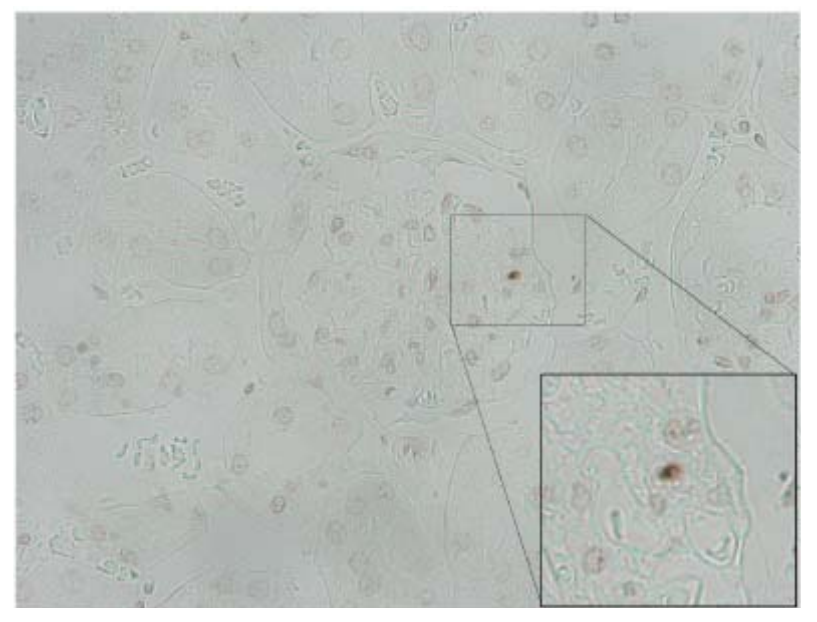

Figure 4. Apoptotic cell in KK-A $\mathrm{A}^{y}$ mouse $(\times 400)$. One apoptotic cell was observed in only one out of 30 glomeruli in KK$\mathrm{A}^{y}$ mice. segmental sclerosis and homogenous PAS-positive hyaline caps. Mean podocyte number per glomerulus (NG pod) in diabetic KK-A ${ }^{y}$ mice was significantly lower than that in non-diabetic BALB/cA mice. Mean NG pod/ GA per glomerulus was also significantly decreased in diabetic KK-A $\mathrm{A}^{y}$ mice. In immunofluorescence, a decrease of WT-1 positive cells (podocytes) in glomeruli was observed in diabetic KK- $\mathrm{A}^{y}$ mice. The level of urinary albumin/creatinine ratio (ACR) in diabetic KK- $\mathrm{A}^{y}$ mice was significantly higher than that in non-diabetic BALB/ cA mice at 20 weeks of age. We previously reported that NG pod was negatively correlated with urinary protein excretion in patients with IgA nephropathy [4]. The general mechanisms of podocyte loss are considered as follows: detachment of intact podocytes from the GBM due to necrosis, apoptosis and/or autophagy [17]. In this study, apoptotic cells in glomerulus were examined in diabetic KK- $\mathrm{A}^{y}$ mice using TUNEL staining. An apoptotic cell was observed only one out of 30 glomeruli in KK- $\mathrm{A}^{y}$ mice. In human diabetic nephropathy, there is no evidence of necrosis and no direct evidence for apoptosis as a major mechanism of podocyte loss. However, apoptosis of podocytes has been determined in cell cultures $[18,19]$. Gu et al. reported that monocyte chemoattractant protein-1 (MCP-1) was induced by advanced glycation end-products and carboxymethllysine (CML) in differentiated podocytes [20]. It is speculated that MCP-1/ cysteine-cysteine chemokine receptor 2 (CCR2) system is involved in the apoptosis of podocytes in diabetic nephropathy. The detachment of intact podocytes from the GBM has been reported in Japanese diabetic patients [21]. Nakamura et al. [21] indicated that podocytes in the urine may be a useful marker of disease activity in patients with diabetic nephropathy. The excretion of podocytes into the urine (podocyte loss) and subsequent hypertrophy may reflect disease activity in glomerular disease $[4,22]$. Podocytes in adults did not undergo mitosis. Normally the only way to respond to injury is by cell hypertrophy. Thus, increased podocyte surface area reflects the extent of podocyte hypertrophy. Since the extent of podocyte hypertrophy is limited, segments of the GBM become denuded in severe cases. Direct contact of the naked portion of the GBM with the parietal epithetlium of Bowman's capsule might lead to adhesion to Bowman's capsule and potential segmental sclerosis [23, 24]. It appears that podocyte loss, i.e. podocytopenia, might induce albuminuria in diabetic KK-A $\mathrm{A}^{y}$ mice. This finding confirmed our previous report that KK- $\mathrm{A}^{y}$ mice, especially in terms of histological findings, are a suitable animal model for glomerular injury in human type 2 diabetic nephropathy.

\section{CONCLUSION}

The results of the present study indicated that podo- 
cyte loss might induce albuminuria in $\mathrm{KK}-\mathrm{A}^{\mathrm{y}}$ mice. This finding confirmed our previous report that $\mathrm{KK}-\mathrm{A}^{y}$ mice, especially in terms of histological findings, are a suitable animal model for glomerular injury in type 2 diabetic nephropathy.

\section{REFERENCES}

[1] Rudberg, S. and Osterby, R. (1998) Diabetic glomerulopathy in young IDDM patients, preventive and diagnostic aspects. Hormone Research, 50, 17-22. doi:10.1159/000053097

[2] Osterby, R. (1992) Glomerular structural changes in type 1 (insulin-dependent) diabetes mellitus: Causes, consequences, and prevention. Diabetologia, 35, 803-812. doi:10.1007/BF00399925

[3] Osterby, R. (1996) Lessons from kidney biopsies. Diabetes/Metabolism Reviews, 12, 151-174. doi:10.1002/(SICI)1099-0895(199610)12:3<151::AID-D MR162>3.0.CO;2-1

[4] Hishiki, T., Shirato, I., Takahashi, Y., Funabiki, K., Horikoshi, S. and Tomino, Y. (2001) Podocyte injury predicts prognosis in patients with IgA nephropathy using a small amount of renal biopsy tissue. Kidney \& Blood Pressure Research, 24, 99-104. doi:10.1159/000054214

[5] Lemley, K.V., Lafayette, R.A., Safai, M., Derby, G., Blouch, A., Squarer, A. and Myers, B.D. (2002) Podocytopenia and disease severity in IgA nephropathy. Kidney International, 61, 1475-1485. doi:10.1046/j.1523-1755.2002.00269.x

[6] Macedo, C.S., Lerco, M.M., Capelletti, S.M., Silva, R.J., Pinheiro, D.O. and Spadella C.T. (2007) Reduction of podocytes number in late diabetic alloxan nephropathy: Prevention by glycemic control. Acta Cirurgica Brasileira, 22, 337-341. doi:10.1590/S0102-86502007000500003

[7] Kondo, K., Nozawa, K., Tomita, T. and Ezaki, K. (1957) Inbred strains resulting from Japanese mice. Bulletin of the Experimental Animals, 6, 107-112.

[8] Taketomi, S., Ikeda, H., Ishikawa, E. and Iwatsuka, H. (1982) Determination of overall insulin sensitivity in diabetic mice, KK. Hormone and Metabolic Research, 14, 14-18. doi:10.1055/s-2007-1018909

[9] Matsuo, T. and Shino, A. (1972) Induction of diabetic alterations by goldthioglucose-obesity in KK, ICR and C57BL mice. Diabetologia, 8, 391-397. doi:10.1007/BF01212165

[10] Shike, T., Gohda, T., Tanimoto, M., Funabiki, K., Horikoshi, S., Hirose, S., Shirai, T. and Tomino, T. (2005) Chromosomal mapping of a quantitative trait locus for the development of albuminuria in diabetic KK/Ta mice. $\mathrm{Ne}$ phrology Dialysis Transplantation, 20, 879-885. doi:10.1093/ndt/gfh665

[11] Ito, T., Tanimoto, M., Matsumoto, M., Yamada, K., Kaneko, S., Msatsumoto, M., Obayashi, K., Hagiwara, S., Murakoshi, M., Aoki, T., Wakabayashi, M., Gohda, T., Funabiki, K., Maeda, K., Horikoshi, S. and Tomino, Y. (2006) Glomerular changes in the KK- $\mathrm{A}^{\mathrm{y}} / \mathrm{Ta}$ mouse: A possible model for human type 2 diabetic nephropathy. Nephrology, 11, 29-35.

doi:10.1111/j.1440-1797.2006.00543.X

[12] Okazaki, M., Saito, Y., Udaka, Y., Maruyama, M., Murakami, H., Ota, S., Kikuchi, T. and Oguchi, K. (2002) Diabetic nephropathy in KK and KK-A ${ }^{\mathrm{y}}$ mice. Experimental Animals, 51, 191-196. doi:10.1538/expanim.51.191

[13] Weibel, E.R. (1979) Stereological Methods: Practical Methods for Biological Morphometry. Academic Press, Inc., London.

[14] Cohen, M.P. and Ziyadeh, F. N. (1996) Role of amadorimodified nonenzymatically glycated serum proteins in the pathogenesis of diabetic nephropathy. Journal of the American Society of Nephrology, 7, 183-190.

[15] Fukami, K., Ueda, S., Yamagishi, S., Kato, S., Inagaki, Y., Takeuchi, M., Motoyama, Y., Bucala, R., Iida, S., Tamaki, K., Imaizumi, T. and Cooper, M.E. (2004) AGEs activate mesangial TGF-beta-smad signaling via an angiotensin II type I receptor interaction. Kidney International, 66, 2137-2147. doi:10.1111/j.1523-1755.2004.66004.x

[16] Pagtalunan, M.E., Miller, P.L., Jumping-Eagle, S., Nelson, R.G., Myers, B.D., Rennke, H.G., Coplon, N.S. and Meyer, T.W. (1997) Podocyte loss and progressive glomerular injury in type II diabetes. Journal of Clinical Investigation, 99, 342-348. doi:10.1172/JCI119163

[17] Lemley, K.V. (2008) Diabetes and chronic kidney disease: Lessons from the Pima Indians. Pediatric Nephrology, 23, 1933-1940. doi:10.1007/s00467-008-0763-8

[18] Asanuma, K., Campbell, K.N., Kim, K., Faul, C. and Mundel, P. (2007) Nuclear relocation of the nephrin and CD2AP-binding protein dendrin promotes apoptosis of podocytes. Proceedings of the National Academy of Sciences USA, 104, 10134-10139. doi:10.1073/pnas.0700917104

[19] Asanuma, K., Yanagida-Asanuma, E., Takagi, M., Kodama, F. and Tomino, Y. (2007) The role of podocytes in proteinuria. Nephrology, 12, 15-20. doi:10.1111/j.1440-1797.2007.00876.x

[20] Gu, L., Hagiwara, S., Fan, Q., Tanimoto, M., Kobata, M., Yamashita, M., Gohda, T., Ni, Z., Horikoshi, S. and Tomino, Y. (2006) Role of receptor for advanced glycation end-products-induced monocyte chemoattractant protein1 expression in differentiated mouse podocytes. Nephrology Dialysis Transplantation, 21, 299-313. doi:10.1093/ndt/gfi210

[21] Nakamura, T., Ushiyama, C., Suzuki, S., Hara, M., Shimada, N., Ebihara, I. and Koide, H. (2000) Urinary excretion of podocytes in patients with diabetic nephropathy. Nephrology Dialysis Transplantation, 15, 1379-1383. doi:10.1093/ndt/15.9.1379

[22] Hara, M., Yanagihara, T., Takada, T., Itoh, M., Matsuno, M., Yamamoto, T. and Kihara, I. (1998) Urinary excretion of podocytes reflects disease activity in children with glomerulonephritis. American Journal of Nephrology, 18, 35-41. doi:10.1159/000013302

[23] Kretzler, M. (2005) Role of podocytes in focal sclerosis: Defining the point of no return. Journal of the American Society of Nephrology, 16, 2830-2832. 
doi:10.1681/ASN.2005080841

[24] Lemley, K.V., Lafayette, R.A., Safai, M., Derby, G., Blouch, K., Squarer, A. and Myers, B.D. (2002) Podocy- topenia and disease severity in IgA nephropathy. Kidney International, 61, 1475-1485.

doi:10.1046/j.1523-1755.2002.00269.x 